\title{
Multicasting in Heterogeneous Networks
}

\author{
U. Vidya Sagar, U. Naresh
}

\begin{abstract}
Mobile devices, such as smart phones and tablet PCs, are becoming ubiquitous; it is increasingly popular for users to watch online streaming videos from their mobile devices. Because of the nature of wireless transmission, wireless routers (such as access points or base stations), they can get video transmissions from the video server and then send leads to several interested customers. In these videos, however, customers can be placed in different positions, and therefore they can deal with different wireless channels. In addition, customers may be interested in different video content, so different videos may have some popularity. Wireless heterogeneity and user interests make it difficult to break the bandwidth of wireless router video multicast. In this article, we propose a Utility-Based LayerEncoded Multicast Scheme, based on an instrument that takes into account the characteristics of the channels and heterogeneous users. The proposed approach that takes into account interests can improve the average visual quality to $9 \mathrm{db}$.
\end{abstract}

Keywords: multi casting, heterogeneous networks, broad casting, layered based encoding..

\section{INTRODUCTION}

Remote contraptions, for instance, PDAs and tablet PCs have ended up being ubiquitous. With the creation pervasiveness of spilling applications like YouTube and Netflix, a remote switch can help restore video streams from video servers and send them to customers who are amped up for this video. Starting late, customers capably demand video content through remote assistance subordinate in solitude focal core interests. Since various stories are heterogeneous in recognizable quality, the obliged usage of remote information move limit concerning most of the video pictures referenced is a delicate issue for remote blended media.

On the most overpowering remote frameworks, for instance, 802.11 , social occasions can be sent using one of the distinctive available piece rates. The speed used in this story identifies with PHY rates. For example, 802.11a, 6 $\mathrm{Mb} / \mathrm{s}$ proposes speed with BPSK and $1 / 2$ encoding speed. Higher throughput uses higher coding paces and change, which streams various bits per amazing body point; thusly, the proportion of bit bumbles is higher than the lower bit rate in an identical channel state. In a multicast condition, the remote switch can move each camcorder to a fixed piece rate as is commonly said. Before long, in light of heterogeneous customer channel conditions, it is possible that the edge must be moved to a pinch of the customers whose channel conditions are satisfactory. As traffic transmission everything considered has a certifiable slowness imprisonment, the remote switch can use this

Revised Manuscript Received on August 14, 2019.

U. Vidya Sagar, Professor \& HOD, Dept of CSE, St.Johns College of Engineering and Technology, Yerrakota, Yemmiganur, Kurnool, A.P. India. (Email: engg.sagar@gmail.com )

U. Naresh, Professor in Dept of CSE, Malla Reddy Engineering College for Women Maisammaguda, Dhulapally(p), Kompally, Hyderabad, Telangana, India.(Email: naresh26uppara@gmail.com) heterogeneous impetus to control the visual idea of each customer. For example, a switch may pick a higher toll to use a period compelled channel to send more video film; For this circumstance, only a few customers with astonishing channel conditions can suitably get these holders and get noteworthy visual quality.

All things considered, the switch can send significantly increasingly moderate shots, (for instance, base layer basic shots) with the objective that all customers can achieve head video quality. It is thusly difficult to pick the fitting piece rate for each video picture so remote information move purpose of restriction can be used to altogether meet the visual idea of customers. Of course, since customers may require unquestionable video cuts, multicast gives must be confined to an obliged remote information move limit. Switches may be pure to dole out a general bit of information move limit sources to each video stream. In any case, this methodology is unsuitable, in light of the way in which that each video has a substitute number of referencing.

A couple of stories require more customers; as necessities be, the switch can make an increasingly unmistakable if all else fails visual quality overhaul for the remote possibility that it gives constantly radio intermittent source to sending essential video pictures. You may envision that another possible strategy is to distribute information transmission hotspots for records that are in degree to their obvious quality. Even if the two videos have the same number of customers, their customers may have different channel conditions, and video images must be sent at a different speed, thus using different channel times. As a result, the flow selection problem becomes more difficult if heterogeneity is to be considered in the interest of users.

\section{RELATED WORK}

To the degree we could know, the examination field concerning authentication of a framework in heterogeneous remote structures from a point of perspective on multicast transport isn't particularly poor down. We found that past work in the district of versatile multicast pivots around subjects like flawless multicast tree advance in multihop unrehearsed courses of action with [1-2].

Ormond and Murphy [3] propose a structure decision strategy that uses different possible utility inspirations driving constrainment. Their answer is customer driven and does not present any multicast condition. An exchange between different customers and structures isn't considered either. Ormond and Murphy brief that the impact of different customers working in a basically dubious territory

Published By:

Blue Eyes Intelligence Engineering

\& Sciences Publication 


\section{MULTICASTING IN HETEROGENEOUS NETWORKS}

ought to be moreover reviewed.

Gluhak et al. [4] consider the issue of picking the perfect vehicle ways for multicast relationship with social events of heterogeneous pros. The proposed check picks the vehicle route subject to different streamlining targets. In any case, Gluhak et al. address the issue only for the ideal static multicast case without considering customers crossing different cells. Likewise, it requires that the data of the conditions in remote structures and tendencies of gatherers is totally shared. In their work, multicast support does not change during the level of a partnership, and multicast social gatherings are not worked with idea of customers' advancements. Through our eyes, this is truly not a sensible case for remote frameworks. In like manner, the proposed request figuring depends on a standard as appeared by which the beneficiaries are appropriated into two sets: the specialists where only a particular structure is open versus the recipients where a few frameworks are available. The impact of the customers inside the going with set, by uprightness of this passing on, neglected.

Yang and Chen [5] propose an exchange speed fit multicast computation for heterogeneous remote structures that is formed as an Integer Linear Programming issue that is understood using Lagrangian slackening up [6]. The estimation headings making faultless most minimal course trees for multicast parties.

In this structure, monstrous parameters, for instance, costs of affiliation or customer's speed are not considered.Jang et al. [7] present a structure for useful framework resource use in an adaptable multicast condition. This structure is made for heterogeneous frameworks and sees arrange decision subject to structure and terminal attributes and Quality of Service (QoS). Regardless, in the proposed segment, the structure decision is performed thoroughly subject to the terminal's affinities; the framework perspective isn't considered; and the methodology does not upgrade the utilization of structure resources.

Hou et al. [8] propose an awesome multicast organizing plan for blended media benefits in IEEE 802.16 based remote metropolitan zone frameworks (WMAN). The getting dealt with is considered for one base station that further re-sends the data to different supporter stations. These are assembled into different multicast parties and the customers are alloted to the social affairs. The makers consider two outstanding ways to deal with oversee direct select multicast packs for affiliations: the unpredictable decision and the channel state cautious affirmation. The point of view is obliged by the base station and kept to one structure improvement. No structure heterogeneity is considered.

The Multicast Mobility (multimob) working party [9] turns its development around supporting multicast in a valuable space. The essential focal reasons for the gettogether are to work out structures for supporting multicast source flexibility and bits that update multicast traffic during a handover. The party correspondingly reports the technique of IGMPv3/MLDv2 in flexible conditions. In this sense, they broaden the IGMPv3/MLDv2 shows up for use in the solid zone and improve Proxy Mobile IPv6 to coordinate multicast positively. In any case, they don't consider any adjustments transversely over different access structures.

\section{UTILITY-BASED LAYER-ENCODED MULTICAST SCHEME \& RESULTS}

The WiMAX burst profile is a set of codec parameters (reflecting the channel state) that allows the BS to send data to the SS, and each SS must agree its transmission profile with the BS before the connection begins. Various overload profiles provide robustness and transmission speeds at different levels. The stronger the overload profile, the lower the data transfer rate. If the stream is transmitted to a set of users with different transfer profiles, the BS must choose the most powerful transfer profile (that is, the one with the lowest data transfer rate) so that all users can receive the same video quality level. In our system, the base station collects group members based on the user group in order to use them in descending order of the channel conditions and uses the strongest transmission profile from all users selected to receive the data. ship. In fact, the transmission of multiple subscriber streams for those with better channel quality makes it possible to make better use of resources.

To send a multicast application to multiple users, you need to use a stronger switch profile. Therefore, a longer period of time is required to send the program. We classify previous works related to wireless video multicast into two categories: 1) single-rate video multicast, and 2) multi-rate video multicast.

\section{Single-rate Video Multicast:}

[10]Proposed a layered hybrid ARQ scheme to determine whether channel time should be used to transfer more layer yields or send FEC packets to restore packet loss.

Work [11] improves visual quality through network coding to combine packets from different multicast sessions. Another work [12] - [15] focuses on the fixed speed selection for packet transmission. They offer a leader board algorithm that allows multicast resources to efficiently select a rate that can send data to customers who are facing the worst channel status. In this article, we focus on multi-speed video multicasting, which allocates bandwidth resources by transferring a well-selected set of frames at correct bit rates according to the heterogeneity of wireless conditions and user interests.

\section{Multi-rate Video Multicast:}

[16] focuses on each frame rate corresponding to each video frame to better utilize the bandwidth source to optimize overall visual quality. [17], it is proposed to use relay nodes to further improve the visual quality of the multicast multicast video. Our work is based on previous protocols, but our protocol is different, because both wireless conditions and heterogeneity are taken into account in the interests of the users.

\section{Broadcast and Multicast Services}

Multicast and Broadcast Services (MBS) are compatible with the heterogeneous network. Network broadcasting and multicast are two important routing systems used in group communications. In the case of network transmission, the 
source node generates in the alternative, distributed to all nodes in the network, while the multicast packets only form a subset of subsets, creating and maintaining the structure data dissemination. Due to the multicasting overhead costs, sometimes it's more efficient to use broadcasts across the entire network, not multicasting, even if the data is for a subset of nodes and meets the following requirements.

- Flexible distribution of radio resources

- MS low power consumption

- Supports audio and video broadcasts for data transmission.

- Low channel switching time.

\section{Heterogeneous Network}

A heterogeneous structure is made as a mix out of various (wired/remote) sort out degrees of progress for persuading data transparency over the framework. Heterogeneous framework conditions display the exchange off and simultaneousness of various remote access progresses with different show stacks and sponsorship for a level of occupations and affiliations that meet the varying affiliation nuts and bolts that must be met arranged structure resources with different degrees of normal media abilities to find the opportunity to open frameworks. The requirement for heterogeneous structures results from the fundamental for convincing mix in regards to the latest and most settled advances. For example, new upgrades, for instance, HSDPA and WiMAX ought to be yielded to existing advances, for instance, GSM, GPRS. The heterogeneous framework in like manner merges media transmission structures. UTRAN and GERAN dealt with 3GPP frameworks correspondingly as other traditionalist access structures, for instance, 802.11 and WLAN transport developments, likewise as assistance for extended convenientce. The heterogeneous structure of the framework is showed up in Figure 1.

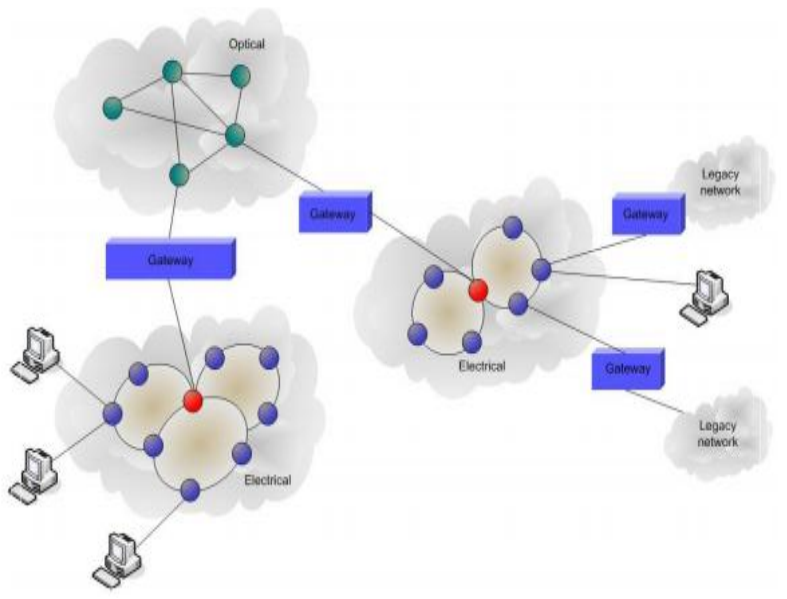

Fig 1. Structure of Heterogeneous Network

\section{Figures and Tables}

Positioning Figures and Tables: Place figures and tables at the top and bottom of columns. Avoid placing them in the middle of columns. Large figures and tables may span across both columns. Figure captions should be below the figures; table heads should appear above the tables. Insert figures and tables after they are cited in the text. Use the abbreviation "Fig. 1", even at the beginning of a sentence.

\section{CONCLUSION}

We have investigated wireless video multicasting due to the heterogeneity of wireless channels and user interests. We formulate a problem with the choice of attached images and the flow of the whole programming model. In order to effectively assign a bandwidth to a limited channel, we propose a utility based video multicast, called boundary value, capable of forwarding the frame at an appropriate speed to increase overall visual quality. Our proposed scheme, which is a bit useful, can improve visual quality by improving the PSNR by $9 \mathrm{~dB}$.

\section{REFERENCES}

1. J. G. Jetcheva, "Adaptable intrigue driven multicast managing in multi-maintain a strategic distance from remote astoundingly doled out frameworks," Ph.D. article, Carnegie Mellon University, Pittsburgh, PA, USA, 2004.

2. J. Yuan, Z. Li, W. Yu, and B. Li, "A cross-layer headwaystructure for multihop multicast in remote work frameworks," IEEE J. Sel. Zones Commun., vol. 24, no. 11, pp. $\underline{2092}-\underline{2103}$, nov. 2006.

3. O. Ormond and J. Murphy, "Utility-based sharp structure decision," in IEEE Int'l Conf. on Communications, ICC, 2006.

4. A. Gluhak, K. Eat, K. Moessner, and R. Tafazolli, "Multicast transporter presentation in heterogeneous remote structures," in IEEE Int'l Conf. on Communications, ICC, vol. 2, May 2005, pp. 13721377.

5. D.- N. Yang and M.- S. Chen, "Skilled resource task for remote multicast," IEEE Transactions on Mobile Computing, vol. 7, no. 4, pp. 387-400, Apr. 2008.

6. M. L. Fisher, "The lagrangian discharging up structure for controlling number programming issues," Manage.Sci., vol. 50, no. 12 Supplement, pp. $1861\{1871$, Dec. 2004.

7. I.- S. Jang, W.- T.Kim, J.- M.Park, and Y.- J. Park, Mobile multicast framework based mih for helpful structure resource use in heterogeneous structures," in Proc. of the twelfth Int'l Conf. on AdvancedCommunication Technology, ser. ICACT'10, 2010, pp. 850-854.

8. F. Hou, L. Cai, P.- H. Ho, X. Shen, and J. Zhang, IA satisfying multicast booking plan formultimedia benefits in ieee 802.16 structures," WirelessCommunications, IEEE Transactions on, vol. 8, no. 3, pp. 1508-1519, $\underline{2009}$.

9. Multicast Mobility Working Group, ICharter forWorking Group," 2010, [Online]. Available:http://datatracker.ietf.org/wg/multimob/suppor t/,got to July $30, \underline{2013}$.

10. Z. Liu, Z. Wu, P. Liu, H. Liu, and Y. Wang, "Layer Bargaining: Multicast Layered Video over Wireless Networks," IEEE J. Sel. Zones Commun., vol. 28, no. 3, pp. 445 - 455, Apr. 2010.

11. H. Seferoglu and A. Markopoulou, "Video-Aware Opportunistic Network Coding over Wireless Networks," IEEE J. Sel. Zones Commun., vol. 27, no. 5, pp. 713 728, Jun. 2009.

12. J. Kuri and S. Kasera, "Strong multicast in multi-get to remote LANs," in IEEE INFOCOM, Mar 1999.

13. A. Basalamah, H. Sugimoto, and T. Sato, "Rate Adaptive Reliable Multicast MAC Protocol for WLANs," in IEEE 
VTC 2006-Spring, 2006.

14. J. Villalon, P. Cuenca, L. Orozco-Barbosa, Y. Seok, and T. Turletti, "Cross-Layer Architecture for Adaptive Video Multicast Streaming Over Multirate Wireless LANs," IEEE J. Sel. Zones Commun., vol. 25, no. 4, pp. 699-711, May 2007.

15. Y. Park, Y. Seok, N. Choi, Y. Choi, and J.- M. Bonnin, "Rate-Adaptive Multimedia Multicasting over IEEE 802.11 Wireless LANs," in IEEE Consumer Communications and Networking Conference, 2006.

16. S. Deb, S. Jaiswal, and K. Nagaraj, "Moving video multicast in wimax structures," in IEEE INFOCOM 2008.

17. K. C.- J. Lin and S.- T.Lee, "Hand-off based Video Multicast with Network Coding in Multi-Rate Wireless Networks," in IEEE GLOBECOM, 2011.

\section{AUTHORS PROFILE}

U. Vidya Sagar, Professor \& HOD, Dept of CSE, St.Johns College of Engineering and Technology, Yerrakota, Yemmiganur

Email: engg.sagar@gmail.com

U. Naresh Professor in Dept of CSE, Malla Reddy Engineering College for Women Maisammaguda, Dhulapally(p), Kompally, Hyderabad, Telangana

Email: naresh26uppara@gmail.com 